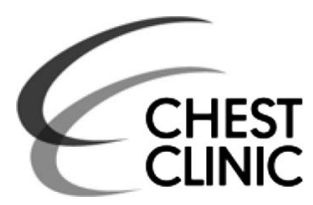

Department of Respiratory Medicine, Cheltenham General Hospital, Cheltenham, Gloucestershire, UK

\section{Correspondence to}

Dr Joseph Hutton, Department of Respiratory Medicine, Cheltenham General Hospital, Sandford Road, Cheltenham, Gloucestershire GL53 7AN, UK; Jh7480@my.bristol.ac.uk

Received 26 May 2014 Revised 2 August 2014 Accepted 14 August 2014 Published Online First

5 September 2014

\title{
A rapid change in a patient's chest radiograph appearances
}

\author{
Joseph Hutton, Claire McBrien, Zafeiris Sardelis, Garrett McGann
}

\section{CLINICAL PRESENTATION}

A 72-year-old man with a history of rheumatoid arthritis (on multiple immunosuppressants: leflunomide, etanercept and hydroxychloroquine) was admitted with headache and fever. He had no other symptoms or relevant medical history. Clinical examination demonstrated coarse crepitations throughout the right upper zone. Chest radiograph revealed consolidation in the right upper zone, peripherally (figure 1A).

The patient's immunosuppressive agents were stopped and he was treated with intravenous benzylpenicillin, clarithromycin and a single dose of gentamicin. After several days of treatment, he improved clinically. Unfortunately 7 days into the admission, he awoke feeling dyspnoeic. He coughed and experienced rightsided pleuritic pain, which resolved following an episode of haemoptysis (a few millilitres only). Clinical examination demonstrated decreased air entry in the right lower zone. A repeat chest radiograph appeared to demonstrate complete resolution of the right upper zone consolidation and new right lower zone consolidation with loss of clarity of the right hemidiaphragm and the right heart border (figure 1B).

The change in the radiographic appearances was so drastic and unexpected; a repeat chest radiograph was ordered to ensure the correct patient had been imaged. The findings were confirmed. CT of the chest was, therefore, requested (figure 2).

\section{QUESTION}

What is the diagnosis?
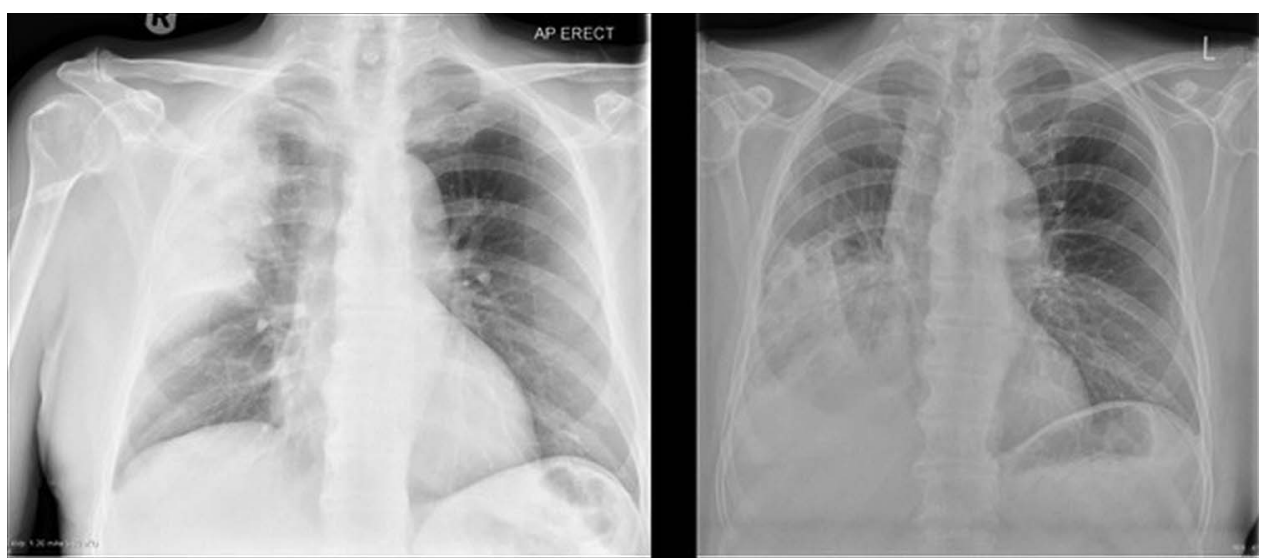

Figure 1 (A) Admission chest radiograph. (B) Repeat chest radiograph following episode of pleuritic chest pain and haemoptysis. 


\section{ANSWER}

The CT demonstrated reversal of the right-sided oblique fissure and an abnormal anterior course of the bronchus intermedius, with rotation of the pulmonary vessels. The right lower lobe pulmonary artery was pulled in an anterior course alongside the bronchus intermedius. There was extensive right-sided consolidation with an associated pleural effusion. Appearances indicated 180 -degree torsion of the right lung (figure 2). The patient was urgently referred to the regional cardiothoracic centre and underwent right upper and lower lobectomies. He was discharged home.

Pulmonary torsion is a rare but life-threatening event, ${ }^{1}$ defined as parenchymal rotation on the bronchovascular pedicle. ${ }^{2}$ This can affect a single lobe or, less frequently, an entire lung. ${ }^{2}$ Torsion most commonly affects the right middle lobe after right upper lobectomy ${ }^{1}$ but can follow other thoracic surgical procedures ${ }^{13}$ or chest trauma. ${ }^{34}$ It very rarely occurs spontaneously. ${ }^{24}$

Pulmonary torsion may compromise airways, arterial supply and venous drainage, risking haemorrhagic infarction and gangrenous necrosis of the affected lung. ${ }^{2}$ In lobar torsion, the torted lobe may expand rapidly due to haemorrhage and infarction. $^{5}$ It constitutes a surgical emergency, requiring urgent exploratory thoracotomy for detorsion or lobectomy. ${ }^{2} 4$

Unfortunately, diagnosis is often delayed. ${ }^{3}$ Possible radiographic features include hilar displacement, rapid lung opacification, bronchial cut-off, bronchial distortion or, as described here, sudden positional change in an opacified lobe. ${ }^{5}$ CT is the investigation of choice.

Risk factors for pulmonary torsion include absence of the inferior pulmonary ligament. ${ }^{1}$ Lobar torsion post-thoracic surgery may be caused by a complete interlobular fissure lacking parenchymal bridging between adjoining lobes. ${ }^{1}$ Such cases may be induced by pathology such as pleural effusion, pneumo-

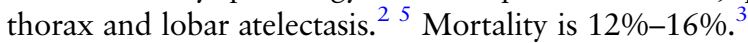

Acknowledgements We are grateful to the patient in question for his kind permission to use his case as a teaching point, to the excellent radiology department at Cheltenham General Hospital and to the Bristol Royal Infirmary for their specialist input.

Contributors $\mathrm{JH}$ planned and carried out the main draft of this work and performed the literature review. He is the main guarantor for its content. CMcB obtained patient consent, proof-read the manuscript, provided further references and was heavily involved in the initial editing process. GMcG proof-read the manuscript, provided the images and reported their content. ZS proof-read the manuscript and offered alterations. He was also the clinical lead on this case and was responsible for the positive outcome in this patient.

Competing interests None.

Patient consent Obtained.

Provenance and peer review Not commissioned; externally peer reviewed.

\section{REFERENCES}

1 Hendriks J, Van Schil P, De Backer W, et al. Massive cerebral infarction after completion pneumonectomy for pulmonary torsion. Thorax 1994;49:1274-5.

2 Irie M, Okumura N, Nakano J, et al. Spontaneous whole-lung torsion after massive pleural effusion and atelectasis. Ann Thorac Surg 2014;97:329-32.

3 Cable DG, Deschamps C, Allen MS, et al. Lobar torsion after pulmonary resection: presentation and outcome. J Thorac Cardiovasc Surg 2001;122:1091-3.

4 Koziarkiewicz M, Taczalska A, Piaseczna-Piotrowska A. Pulmonary torsion as an atypical complication of congenital esophageal atresia repair-a case report and review of literature. J Paediatr Surg 2007;42:E5-9.

5 Felson B. Lung torsion: radiographic findings in nine cases. Radiology $1987 ; 162: 639-43$.

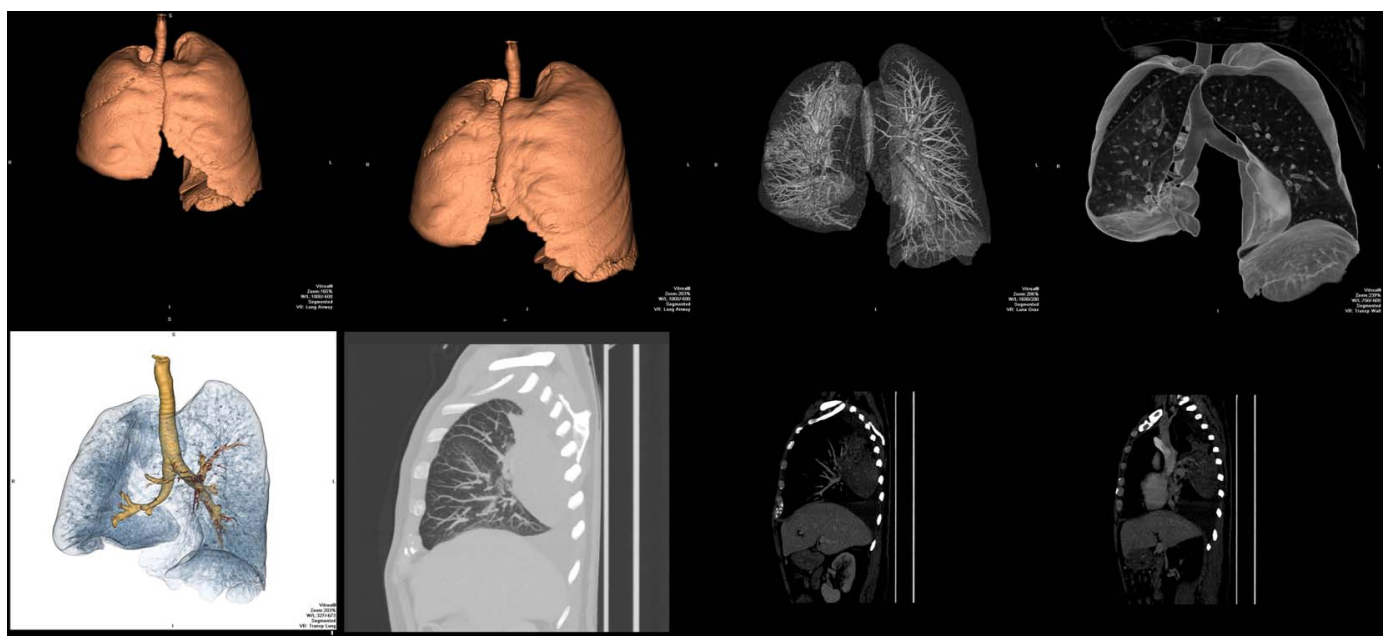

Figure 2 Sagittal sections and three-dimensional-rendered reconstructions of CT scan. Note the reversal of the right oblique fissure. 